\title{
Endoplasmic reticulum stress stimulates the release of extracellular vesicles carrying danger-associated molecular pattern (DAMP) molecules
}

\author{
Gavin P. Collett ${ }^{1}$, Christopher W. Redman ${ }^{1}$, Ian L. Sargent ${ }^{1}$ and Manu Vatish ${ }^{1}$ \\ ${ }^{1}$ Nuffield Department of Women's \& Reproductive Health, University of Oxford, Women's Centre Level 3, John Radcliffe \\ Hospital, Oxford OX3 9DU, UK \\ Correspondence to: Manu Vatish, email: manu.vatish@obs-gyn.ox.ac.uk \\ Keywords: endoplasmic reticulum stress; extracellular vesicles; ER stress; pathology \\ Received: October 06, $2017 \quad$ Accepted: January 02, $2018 \quad$ Published: January 11, 2018 \\ Copyright: Collett et al. This is an open-access article distributed under the terms of the Creative Commons Attribution License \\ 3.0 (CC BY 3.0), which permits unrestricted use, distribution, and reproduction in any medium, provided the original author and \\ source are credited.
}

\section{ABSTRACT}

Disturbances in endoplasmic reticulum (ER) function lead to ER stress which, when severe or prolonged, may result in apoptosis. Severe ER stress has been implicated in several pathological conditions including pre-eclampsia, a multisystem disorder of pregnancy associated with the release of pro-inflammatory factors from the placenta into the maternal circulation. Here, we show that severe ER stress induced by two distinct mechanisms in BeWo choriocarcinoma cells leads to the release of extracellular vesicles (EVs) carrying pro-inflammatory damage-associated molecular pattern (DAMP) molecules. Co-treatment with the antioxidant pyrrolidine dithiocarbamate results in a reduction in ER stress-induced EV-associated DAMP release. We further demonstrate that severe ER stress is associated with changes in the expression of several stress-related proteins, notably Cited-2 and phosphorylated JNK. Together, these data indicate that severe ER stress-mediated release of EVassociated DAMPs may contribute to the heightened systemic maternal inflammatory response characteristic of pre-eclampsia and may also be relevant to other chronic inflammatory diseases which display elevated ER stress.

\section{INTRODUCTION}

Endoplasmic reticulum stress, resulting from disturbances in ER function and the accumulation of unfolded or misfolded proteins in the ER lumen, leads to the unfolded protein response (UPR), a cytoprotective response comprising multiple intracellular signalling pathways [1]. Under mild ER stress conditions, activation of these pathways is sufficient to restore normal ER function and cellular homeostasis. However, if ER stress is severe or prolonged, or if UPR signalling pathways are compromised, then apoptotic cell death takes place. Chronic ER stress, with ensuing cell injury or death, has been implicated in a number of pathological conditions including diabetes [2], atherosclerosis [3] and neurodegenerative diseases [4]. In pregnancy, it has been shown that placentae from cases of intrauterine growth restriction (IUGR) display activation of ER stress pathways and that these are further elevated in IUGR pregnancies complicated with pre-eclampsia (PE), a multisystem disorder of pregnancy characterised by an exacerbated maternal systemic inflammatory response [5]. More recently, it was revealed that activation of placental ER stress response pathways is greater in severe earlyonset PE $(<34$ weeks) than in late-onset disease $(\geq 34$ weeks) [6].

Pre-eclampsia is associated with the release of multiple pro-inflammatory factors from the placental syncytiotrophoblast into the maternal circulation. These factors include extracellular vesicles (EVs) and damage associated molecular pattern molecules (DAMPs). EVs comprise exosomes ( 50-200 nm, released by exocytosis of endosome-derived multivesicular bodies), microvesicles $(\sim 100 \mathrm{~nm}-1 \mu \mathrm{m}$, released by direct budding 
from the plasma membrane) and apoptotic bodies $(\sim 1$ $5 \mu \mathrm{m}$, released by cells undergoing programmed cell death) [7], and have been implicated in both the maintenance of normal pregnancy and the pathology of disorders of pregnancy, particularly pre-eclampsia [8]. DAMPs, also known as alarmins, are highly pro-inflammatory molecules released by stressed or damaged cells which act as endogenous danger signals [9]. They can bind to and activate cells of the innate immune system leading to the initiation or exacerbation of a pro-inflammatory response. Examples of DAMPs include high mobility group box1 (HMGB1), heat shock proteins, S100 calcium-binding proteins, histones and extracellular ATP. Increased serum levels of DAMPs have been associated with inflammatory diseases such as systemic lupus erythematosus [10], atherosclerosis [11] and acute pancreatitis [12]. A role for DAMPS in the pathophysiology of pre-eclampsia is supported by the observation that women with preeclampsia have increased serum levels of several DAMPs including HMGB1 [13], HSP70 [14] and S100B [15]. Furthermore, both HMGB1 [16] and S100B [17] have been shown to be released from trophoblast cells in response to oxidative stress.

Although DAMPs are primarily considered to be soluble molecules, there is growing interest in the notion that DAMP-carrying EVs released from stressed or injured
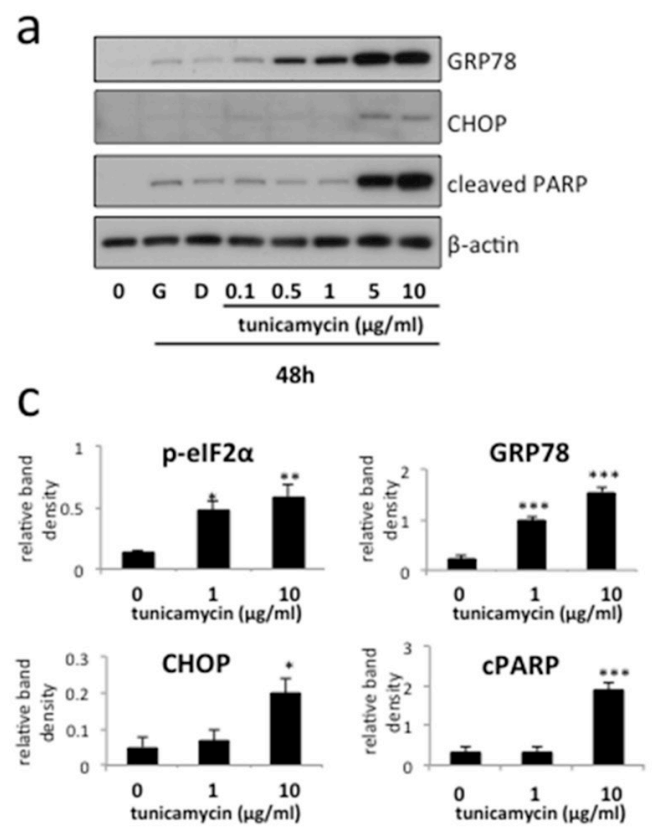

tissues may play a role in the induction or persistence of inflammation [18]. HMGB1 [19], heat shock proteins [20] and ATP [21] have all been shown to be carried in EVs. However, the biological role of EV-associated DAMPs is yet to be ascertained. Whilst it has been demonstrated that palmitate-induced ER stress in hepatocytes stimulates the release of EVs [22], it is unclear whether ER stress can lead to the release of EVs carrying DAMPs. In this study we show that severe ER stress generated by two distinct pathways in BeWo choriocarcinoma cells (a cell line used to model trophoblast) results in the release of EVassociated DAMPs. We propose that this is a mechanism which may contribute to the clinical symptoms of preeclampsia and other chronic inflammatory diseases.

\section{RESULTS}

\section{Induction of mild and severe ER stress by tunicamycin in BeWo cells}

Mild ER stress activates the unfolded protein response. However, if it is severe or prolonged ER stress results in cell death. To mimic these effects in vitro, we treated BeWo cells with increasing doses of tunicamycin, a compound routinely used to induce ER stress (Figure 1a). Immunoblotting revealed a dose-dependent upregulation

\section{b}

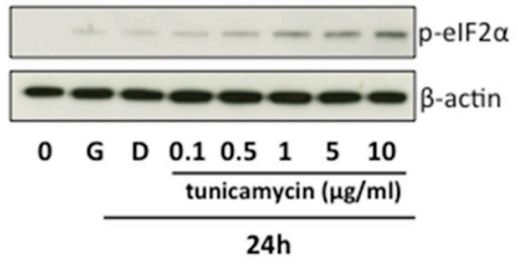

d

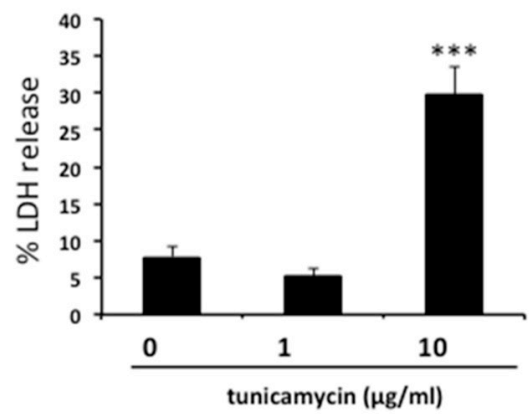

Figure 1: Induction of mild and severe ER stress by tunicamycin in BeWo cells. BeWo cells were treated with growth medium alone (G), vehicle (DMSO) or the indicated concentrations of tunicamycin for 48h (a) or 24h (b). Cell lysates were analysed for the expression of GRP78, phospho-eIF2 $\alpha$, CHOP, cleaved PARP and $\beta$-actin by immunoblotting. Representative blots from three separate experiments are shown. (c) Blots were analysed by densitometric image analysis (Image J), normalising to $\beta$-actin, for GRP78, phosphoeIF $2 \alpha$, CHOP \& cleaved PARP. Results are represented as mean \pm SEM for three separate experiments. ${ }^{*} \mathrm{p}<0.05,{ }^{* *} \mathrm{p}<0.01,{ }^{* * *} \mathrm{p}<0.001$ compared with control (ANOVA). (d) Cells were treated with $1 \mu \mathrm{g} / \mathrm{ml}$ or $10 \mu \mathrm{g} / \mathrm{ml}$ tunicamycin for $48 \mathrm{~h}$, representing mild and severe ER stress respectively. Cell death was analysed by release of lactate dehydrogenase (LDH) into the culture medium. Results are represented as mean \pm SEM for four separate experiments. ${ }^{* * *} \mathrm{p}<0.001$ compared with control (ANOVA). 
of the chaperone protein GRP78 and phosphorylated eIF2 $\alpha$, signifying induction of an ER stress response, but only higher doses $(5-10 \mu \mathrm{g} / \mathrm{ml})$ resulted in increased CHOP expression and PARP cleavage, signifying induction of more severe ER stress resulting in cell death (Figures 1a \& 2a). Accordingly, for further experiments, tunicamycin concentrations of 1 and $10 \mu \mathrm{g} / \mathrm{ml}$ were therefore selected to represent conditions of mild (significant upregulation of GRP78 \& phosphorylated eIF2 $\alpha$ but not CHOP \& cleaved PARP, Figure 1c) and severe (significant upregulation of GRP78, phosphorylated eIF2 $\alpha$, CHOP \& cleaved PARP, Figure 1c) ER stress respectively. Using these conditions we also observed significant increase of LDH release into the culture medium following severe ER stress induction, confirming the induction of cell death in response to severe, but not mild, ER stress (Figure 1d).

\section{Severe ER stress stimulates the release of extracellular vesicles carrying danger-associated molecular pattern (DAMP) molecules}

To determine the effect of ER stress on EV release we subjected BeWo cells to mild or severe ER stress and assessed EV number and size by nanoparticle tracking analysis. This revealed a significant increase in the number of EVs released in response to severe, but not mild, ER stress (Figure 2a). Furthermore, the mean size of vesicles released from severely ER-stressed cells was significantly greater than those shed from unstressed or mildly ER-stressed cells (Figure 2b). Assessment of isolated EVs for expression of damage-associated molecular patterns (DAMPs) by immunoblotting revealed high levels of EV-associated high mobility group protein B1 (HMGB1), heat shock protein 70 (hsp70) and histone H3 released from severely ER-stressed cells whereas these were low or undetectable from unstressed or mildly ER-stressed cells (Figure 2c). In contrast, expression of exosome markers syntenin and alix was detectable in EVs released from unstressed cells but was not increased in response to severe ER stress. It should be noted that these immunoblots were designed to detect the relative amounts of EV-associated DAMPs released into the culture medium and therefore equal volumes of the resuspended EV pellets were loaded. Since these would be expected to contain different amounts of protein (due to differing numbers of EV present) a loading control was not used. Further characterisation of EVs released from severely ER-stressed cells was carried out using iodixanol

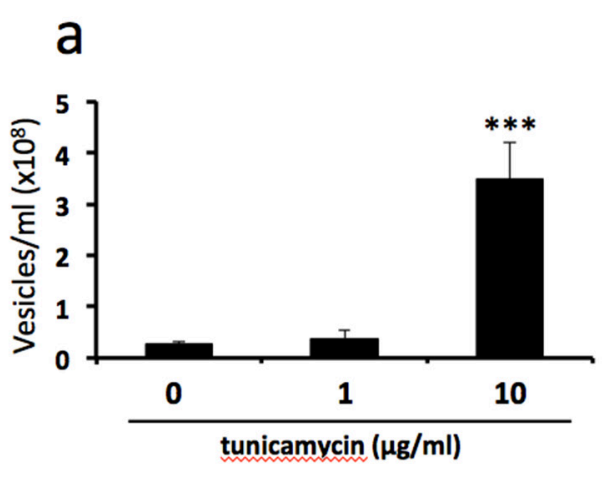

b
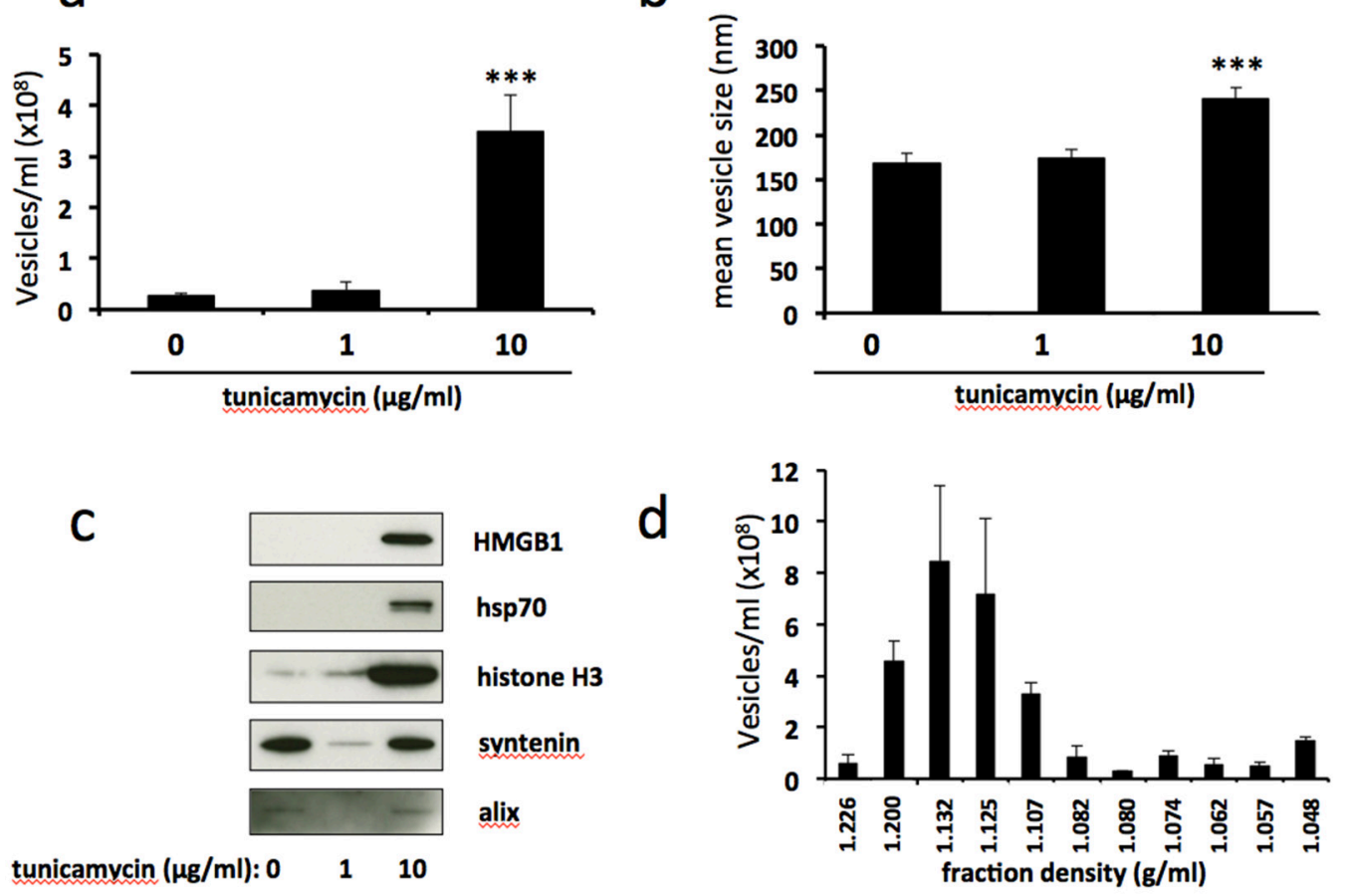

Figure 2: Severe ER stress stimulates the release of extracellular vesicles carrying danger-associated molecular pattern (DAMP) molecules. BeWo cells were treated with $1 \mu \mathrm{g} / \mathrm{ml}$ or $10 \mu \mathrm{g} / \mathrm{ml}$ tunicamycin, or DMSO control, for $48 \mathrm{~h}$. Extracellular vesicles were isolated from the culture medium by ultracentrifugation and analysed by NTA for quantity (a) and size (b). Equal volumes of EV pellet suspensions were analysed for expression of DAMPs HMGB1, hsp70 and histone H3, and exosome markers syntenin and alix by immunoblotting (c). (d) EV pellets from severely ER-stressed cells were resuspended in PBS (500 $\mu 1)$ and overlaid onto 5-40\% discontinuous iodixanol gradients. Ultracentrifugation was carried out at $150,000 \mathrm{xg}$ (maximum) for $18 \mathrm{~h}$ at $4^{\circ} \mathrm{C}$. Fractions $(12 \mathrm{x} 1 \mathrm{ml}) \mathrm{were}$ collected manually and EV concentrations were analysed by NTA. Results are represented as mean \pm SEM for six (a-c) or three (d) separate experiments. ${ }^{* * *} \mathrm{p}<0.001$ compared with control (ANOVA). 
density gradient centrifugation followed by NTA. In iodixanol gradients exosomes localise to fractions with a buoyant density of $1.09-1.11 \mathrm{~g} / \mathrm{ml}[23,24]$. We found only a small proportion $(\sim 11 \%)$ of EVs in the fraction corresponding to this density (Figure $2 \mathrm{~d}$ ). The majority of EVs localised to fractions with higher densities (1.131.2), indicating a predominance of microvesicles and/or apoptotic bodies.

\section{Antioxidant treatment attenuates ER stress- induced release of extracellular vesicles}

ER stress is associated with increased production of reactive oxygen species (ROS) and the development of oxidative stress [25]. Therefore we used the ROSscavenging antioxidant pyrrolidine dithiocarbamate (PDTC) to examine the role of oxidative stress on ER stress-induced release of EVs. Treatment with PDTC significantly reduced the number of EVs released in response to severe ER stress (Figure 3a), and reversed the ER-stress induced increase in mean vesicle size (Figure $3 b)$. Furthermore, release of vesicle-associated HMGB1 and hsp70 was completely abolished by PDTC treatment (Figure 3c). Taken together, these results show that antioxidant treatment attenuates ER stress-induced release of EVs.
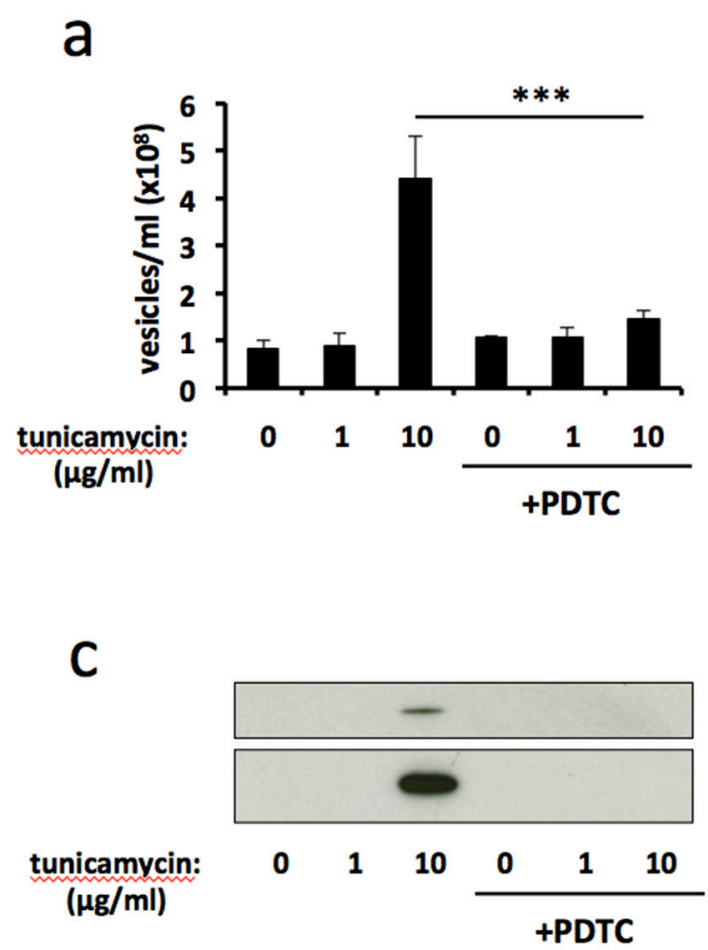

\section{Thapsigargin-induced severe ER stress stimulates release of EV-associated DAMPs}

Tunicamycin induces ER stress by inhibiting $\mathrm{N}$-linked glycosylation, leading to an accumulation of unfolded or misfolded proteins in the ER lumen. In order to assess whether ER stress induced by a different mechanism results in similar release of EV-associated DAMPs, we used thapsigargin, a compound which induces ER stress by disruption of calcium homeostasis. Treatment of BeWo cells with $10 \mu \mathrm{M}$ thapsigargin resulted in upregulation of CHOP expression and the induction of PARP cleavage (Figure 4a). This was accompanied by a significant increase in both number (Figure $4 \mathrm{~b}$ ) and size (Figure 4c) of shed EVs. Immunoblotting revealed that these EVs carried DAMPs HMGB1, hsp70 and histone H3 (Figure 4d). These results show that, as does tunicamycin treatment, severe ER stress induced by thapsigargin stimulates the release of EV-associated DAMPs.

\section{Regulation of stress-related proteins during ER stress}

In order to gain an insight into the pathways involved in ER stress-stimulated EV release in BeWo

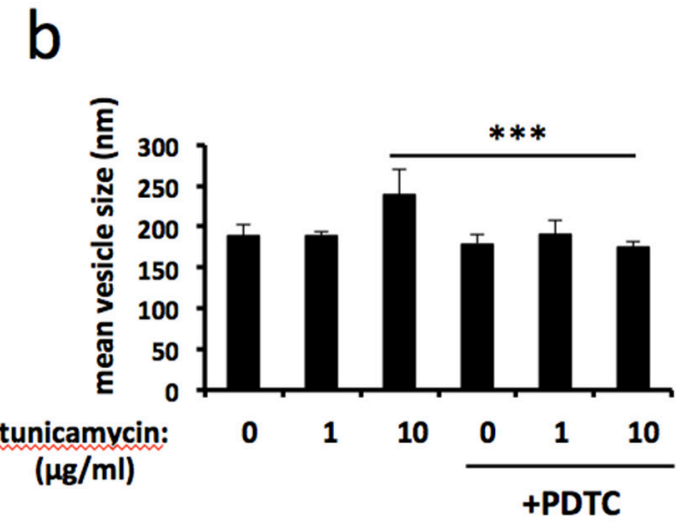

hsp70

HMGB1

Figure 3: Antioxidant treatment attenuates ER stress-induced release of extracellular vesicles. BeWo cells were treated with $1 \mu \mathrm{g} / \mathrm{ml}$ or $10 \mu \mathrm{g} / \mathrm{ml}$ tunicamycin, or DMSO control, for $48 \mathrm{~h}$ in the presence or absence of the ROS scavenging antioxidant pyrrolidine dithiocarbamate (PDTC). Extracellular vesicles were isolated from the culture medium by ultracentrifugation and analysed by NTA for quantity (a) and size (b). Equal volumes of EV pellet suspensions were analysed for expression of HMGB1 and hsp70 by immunoblotting (c). Results are represented as mean $\pm \mathrm{SEM}$ for three separate experiments. ${ }^{* * *} \mathrm{p}<0.001$ compared with control (ANOVA). 
a

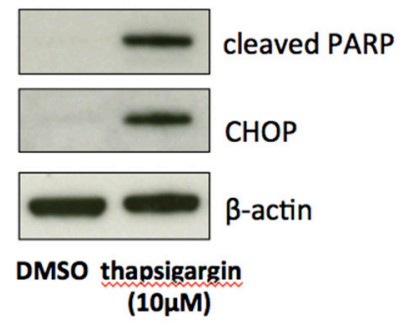

C

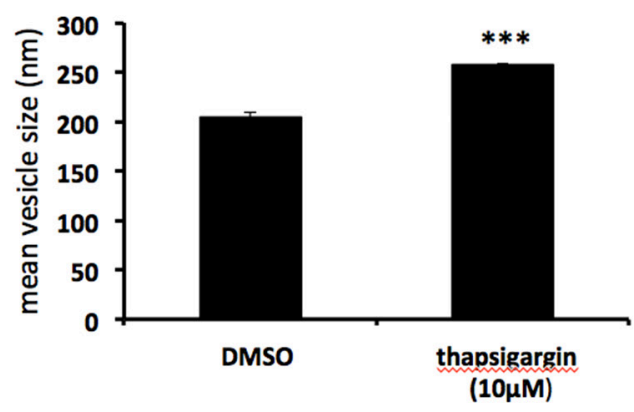

b

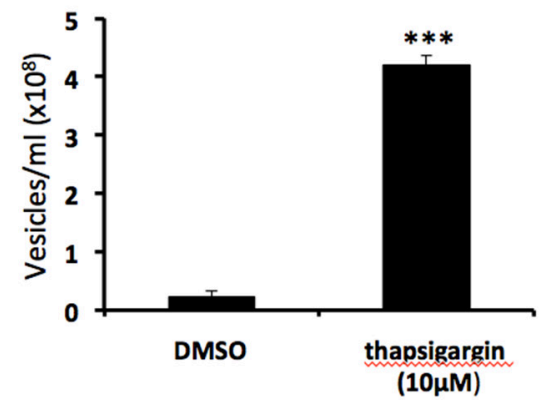

d

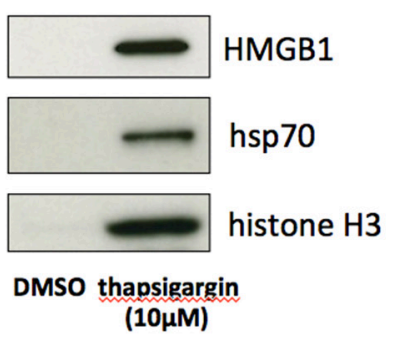

Figure 4: Thapsigargin-induced severe ER stress stimulates release of EV-associated DAMPs. BeWo cells were treated with $10 \mu \mathrm{M}$ thapsigargin or DMSO control for $48 \mathrm{~h}$. Cell lysates were analysed for the expression of CHOP, cleaved PARP and $\beta$-actin by immunoblotting (a). Extracellular vesicles were isolated from the culture medium by ultracentrifugation and analysed by NTA for quantity (b) and size (c). Equal volumes of EV pellet suspensions were analysed for expression of HMGB1, hsp70 and histone H3 by immunoblotting (d). Results are represented as mean \pm SEM for three separate experiments. ${ }^{* * *} \mathrm{p}<0.001$ compared with control (Students $\mathrm{t}$ test).

a

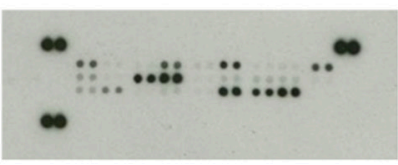

DMSO

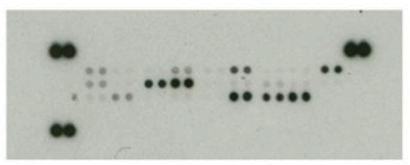

$1 \mu \mathrm{g} / \mathrm{ml}$ tunicamycin

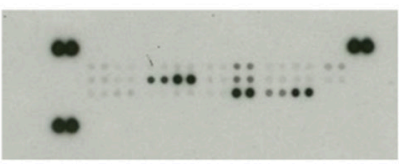

$10 \mu \mathrm{g} / \mathrm{ml}$ tunicamycin

b

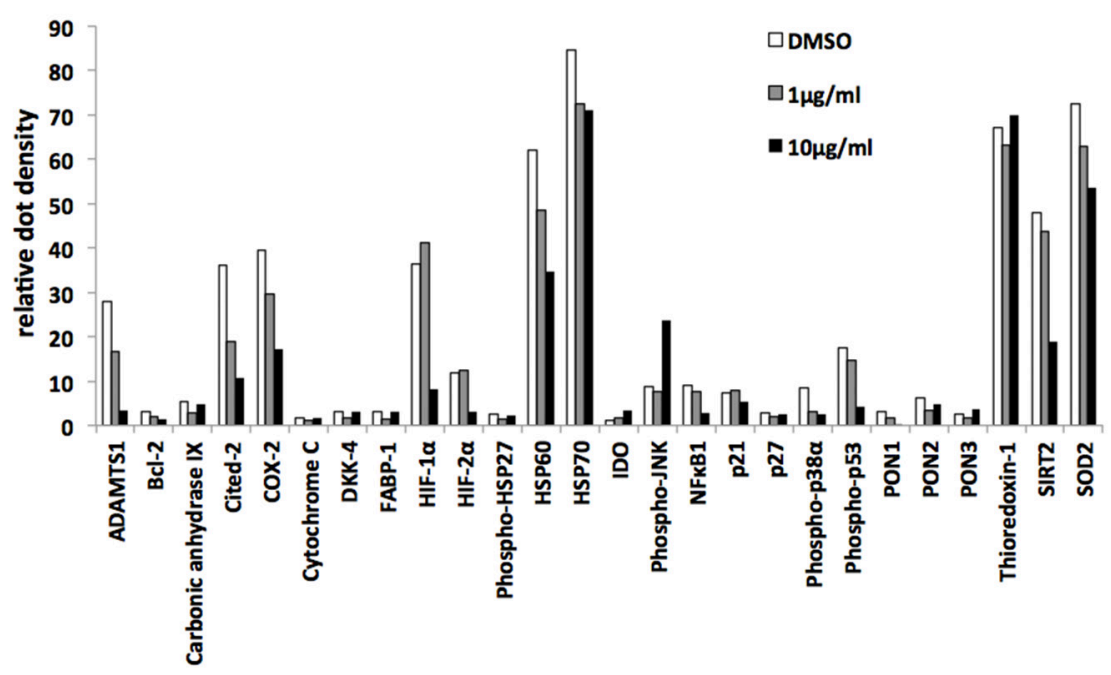

Figure 5: Human cell stress arrays. BeWo cells were treated with $1 \mu \mathrm{g} / \mathrm{ml}$ or $10 \mu \mathrm{g} / \mathrm{ml}$ tunicamycin, or DMSO control, for $48 \mathrm{~h}$. (a) Cell lysate samples containing equal amounts of protein were analysed on human cell stress arrays. (b) The blots shown in (A) were analysed by densitometric image analysis (Image $\mathrm{J}$ ). The graph displays the proteins in the order on the dot blots, from top left to bottom right excluding the three positive control pairs of dots on the top left, top right and bottom left. 
cells we used a human cell stress array. The expression of 26 cell stress-related proteins was analysed in lysates prepared from BeWo cells subjected to tunicamycininduced mild or severe ER stress, or DMSO control, for 48h (Figure 5a). Although not quantitative, densitometric analysis of the developed arrays indicates trends of protein expression in response to ER stress (Figure $5 b)$. Several proteins were downregulated in response to severe ER stress, most notably ADAMTS1, Cox-2, Cited-2, HIF-1 $\alpha$ and SIRT2. One protein, phospho-JNK, was upregulated by severe ER stress. We then used immunoblotting to validate the changes in expression of two of these proteins, Cited-2 and phospho-JNK. In these experiments lysates were made at $24 \mathrm{~h}$ as well as $48 \mathrm{~h}$. Cited-2 was significantly downregulated by severe ER stress at both $24 \mathrm{~h}$ and $48 \mathrm{~h}$ compared with vehicle control (Figures 6a \& 6b). Mild ER stress had no significant effect on Cited-2 expression. Phospho-JNK levels were significantly increased by severe ER stress at both $24 \mathrm{~h}$ and $48 \mathrm{~h}$ but unchanged by mild ER stress (Figures $6 \mathrm{c} \&$ $6 \mathrm{~d})$. These results suggest that downregulation of Cited-2 expression and increased JNK phosphorylation may be involved in the mechanism of ER stress-induced release of EV-associated DAMPs.

a

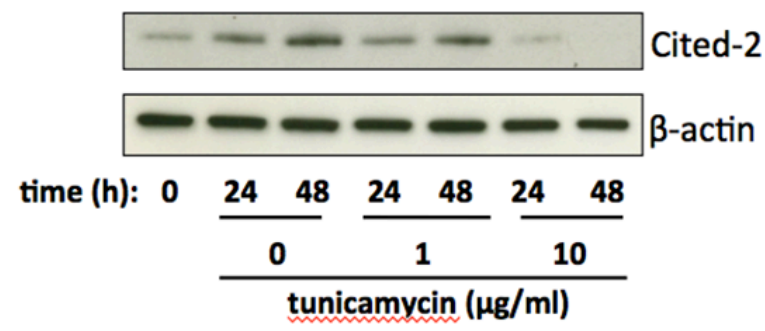

b

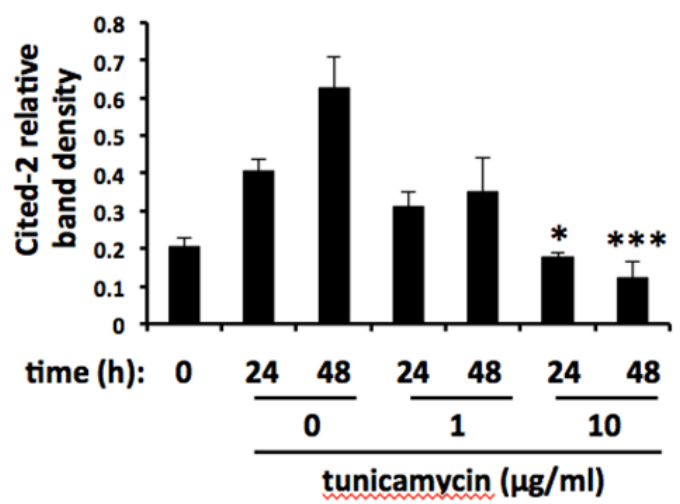

\section{DISCUSSION}

In this study we demonstrate for the first time that severe ER stress leads to the release of EV-associated DAMPs from BeWo cells. We propose that their release from the placenta through this mechanism may contribute to the exacerbated maternal systemic inflammatory response characteristic of pre-eclampsia (Figure 7).

We have used BeWo choriocarcinoma cells as a model of human trophoblast in this study. Although trophoblast cell lines have some limitations, BeWo cells have been used in previous studies to investigate the release of trophoblast-derived EVs [26, 27]. Furthermore, isolation of primary cytotrophoblasts from placental tissue is associated with the activation of ER stress pathways ( $G$ Collett, unpublished observations) which may render these cells inappropriate for the investigations described here. Similarly, we chose to use non-syncytialised BeWo cells for this study since syncytialisation of these cells in culture is associated with decreased cell viability and apoptosis [28] and the activation of ER stress pathways (G Collett, unpublished observations).

Pre-eclampsia is associated with increased placental stress, including activation of ER stress pathways. We

C
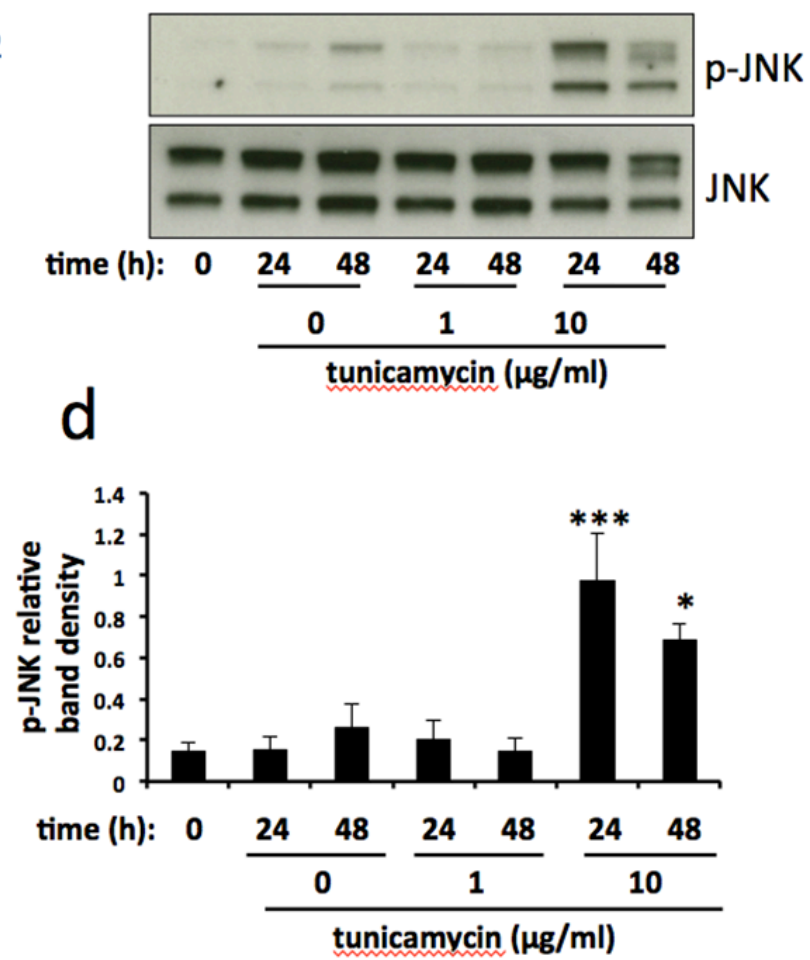

Figure 6: Cited-2 and phospho-JNK expression is altered in severe ER stress. BeWo cells were treated with $1 \mu \mathrm{g} / \mathrm{ml}$ or $10 \mu \mathrm{g} /$ $\mathrm{ml}$ tunicamycin, or DMSO control, for $24 \mathrm{~h}$ and $48 \mathrm{~h}$. At each time point cell lysates were made and analysed for the expression of Cited-2 (a) and phospho-JNK (c). Blots were analysed by densitometric image analysis (Image J), normalising to $\beta$-actin for Cited-2 (b) and total JNK for phospho-JNK (d). Results are represented as mean \pm SEM for three separate experiments. ${ }^{*} \mathrm{p}<0.05,{ }^{* * *} \mathrm{p}<0.001$ compared with control (ANOVA). 
have demonstrated that severe ER stress, induced by two different mechanisms and resulting in $\mathrm{CHOP}$ upregulation and cell death, results in the production of EVs carrying pro-inflammatory DAMPs, whereas mild ER stress does not. These findings are consistent with the hypothesis that low levels of placental ER stress result in reduced cell proliferation and a growth restricted phenotype whereas higher levels of ER stress lead to activation of pro-inflammatory pathways characteristic of preeclampsia [29]. Increased shedding of EVs as a result of cellular stress has been reported previously. For example, oxidative stress increases exosome secretion from retinal pigment epithelial cells whilst adipocytes exposed to lipotoxic stress show enhanced release of extracellular vesicles [30]. Furthermore, ER stress stimulates the release of pro-inflammatory EVs from hepatocytes [22]. Our results show that, in common with most cell types, unstressed BeWo cells constitutively release exosomes that express exosome markers alix and syntenin and carry low or undetectable levels of DAMPs. However, following severe ER stress the observed increase in released EVs is not accompanied by a concomitant increase in EVassociated alix and syntenin expression. Thus it is likely that the EVs shed in response to severe ER stress are predominantly microvesicles and/or apoptotic bodies. This is supported by two further findings: only a small minority of EVs from severely ER-stressed cells localise during density gradient centrifugation to fractions with a buoyant density characteristic of exosomes, and the mean size of EVs released by severely ER-stressed cells is significantly larger than those released by unstressed cells. In addition these vesicles carry high levels of DAMPs HMGB1, hsp70 and histone H3. Increased release of DAMPs has been implicated in the pathogenesis of pre-eclampsia. Circulating levels of HMGB1 [13, 31], hsp70 [32], extracellular ATP [33], S100B [15, 34] and mitochondrial DNA [35] are significantly elevated in women with preeclampsia whilst HMGB1 mRNA and protein expression are increased in severe pre-eclamptic placentas [33]. In addition, HMGB1 released from cultured trophoblast cells following hypoxic stress increases endothelial cell permeability, a feature of pre-eclampsia [16]. Extensive research has highlighted the role of DAMPs in sterile inflammation but it is becoming clear that these molecules can be released from cells in or on EVs as well as in soluble form. For example, HMGB1 is highly concentrated within apoptotic vesicles [19] whilst hsp70 has been shown to be released in a vesicular form which can activate macrophages [36]. Whether EV-associated DAMPs have different functions to those released in soluble form remains to be determined, although vesicular hsp70 is at least 260 -fold more effective in inducing TNFalpha production from macrophages than free recombinant hsp70 [36]. Interestingly, release of DAMPs, including histones and hsp family members, from apoptotic bodies through limited membrane permeabilisation prior

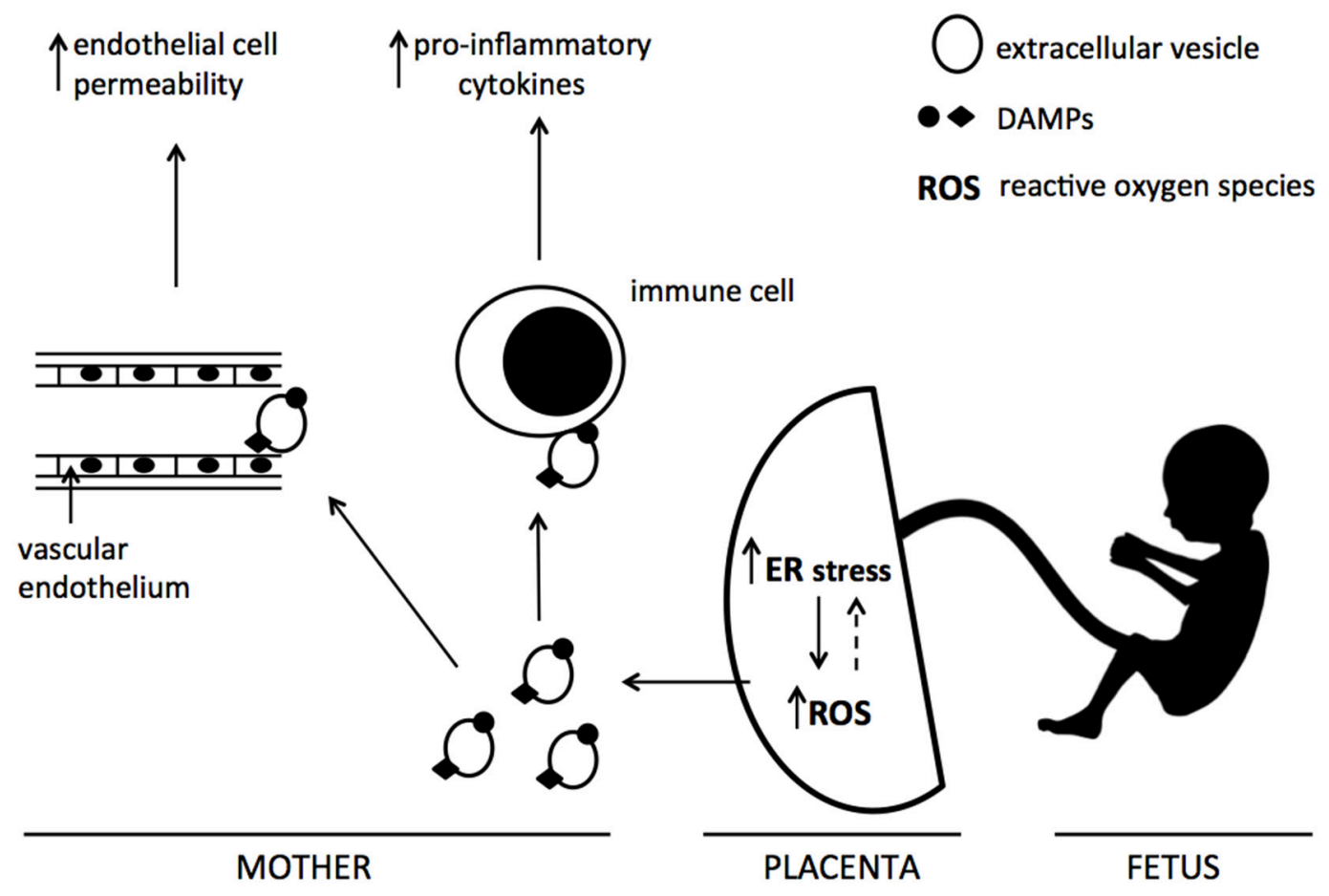

Figure 7: Proposed mechanism of action of ER stress-induced EV-associated DAMPs. Increased placental ER stress, via the production of reactive oxygen species, leads to the release of extracellular vesicles carrying DAMPs into the maternal circulation. These EV-associated DAMPs may interact with maternal endothelial and immune cells, resulting in exacerbated endothelial cell permeability and pro-inflammatory cytokine production. 
to secondary necrosis has been observed, raising the possibility of localised release of pro-inflammatory factors by this EV-mediated mechanism during the early phase of apoptosis [37].

The molecular mechanism by which ER stress leads to the release of EV-associated DAMPs is yet to be elucidated. The endoplasmic reticulum maintains an oxidising environment which facilitates disulphide bond formation and normal protein folding [38]. However, ER stress is associated with the excessive production of reactive oxygen species (ROS) within the ER lumen, leading to oxidative stress and subsequent cell injury and/or death [39]. In accordance with this, our results using a ROS scavenging antioxidant, PDTC, showed complete abrogation of severe ER stress-induced EV release, suggesting that these effects of ER stress are mediated through the generation of ROS and oxidative stress. However, these results may not necessarily reflect a simple, linear upregulation of ROS following ER stress since the relationship between ER and oxidative stress is complex, with ROS playing a role both upstream and downstream of UPR signalling [27], potentially through the establishment of a positive feedback loop [40]. For further mechanistic investigation, we used a human cell stress array to identify proteins showing expression changes in cells undergoing severe ER stress. Cited-2 (CBP/p300-interacting transactivator with Glu/Asprich carboxy-terminal domain 2), a multifunctional transcriptional co-regulator with antiapoptotic properties [41], was significantly downregulated in severely ER stressed cells. A crucial role for Cited-2 in placental function is demonstrated by work showing that its loss in mouse syncytiotrophoblasts results in placental malformation and significantly reduced embryonic growth [42], although whether induction of ER stress plays a role in this is not known. Repression of Cited-2 expression has been shown to induce ER stress in neural stem cells [43] via a mechanism involving upregulation of miR-200b. Therefore, it is tempting to speculate that downregulation of this protein may be a key player in the induction of ER stress and subsequent release of EVs from trophoblast cells. However, it should be noted that Cited-2 expression is increased in culture under control (vehicle only) conditions. This may be due to increasing cell density, which has been shown to alter the expression of other proteins in culture $[44,45]$. Thus it is possible that the growth inhibitory effect of ER stress may be responsible for the relative downregulation of Cited-2 expression compared with control; therefore we cannot rule out the possibility that Cited- 2 may itself be regulated by ER stress rather than vice versa. These themes will be explored in future work.

Whilst most proteins showing a change in expression in ER stressed cells were downregulated, we found a significant elevation of phosphorylated JNK levels. JNK, which is activated by phosphorylation, has been shown to regulate the transition from adaptive to $\mathrm{CHOP}$-dependent apoptotic UPR [46]. This is in agreement with our finding that severe, but not mild, ER stress resulted in a significant increase in JNK phosphorylation. JNK has been implicated in the release of HMGB1 [47]. In particular, JNK mediates free cholesterol-induced apoptosis, necrosis and secretion of HMGB1 in hepatocytes [48]. Thus it is likely that activation of JNK plays a role in the severe ER stress-induced apoptosis and release of EV-associated HMGB1 and other DAMPS which we have observed in this study.

In conclusion, our results suggest that severe ER stress-mediated release of EV-associated DAMPs may possibly contribute to the heightened systemic maternal inflammatory response and endothelial cell permeability characteristic of pre-eclampsia. These results may also be pertinent to other chronic inflammatory diseases which show elevated ER stress. Multiple mechanisms have been demonstrated by which ER stress can promote inflammation in these conditions, including delivery of danger signals to antigen presenting cells following ER stress-induced apoptosis [49]. Therefore it is conceivable that these effects may, in part, be mediated through the release of EV-associated DAMPs.

\section{MATERIALS AND METHODS}

\section{Cell culture}

BeWo cells were obtained from the European Collection of Cell Cultures (Porton Down, UK) and cultured in full growth medium (Dulbecco's modified Eagle's medium/Ham's F12 supplemented with 2 $\mathrm{mM}$ l-glutamine, $100 \mathrm{IU} / \mathrm{ml}$ penicillin, $100 \mu \mathrm{g} / \mathrm{ml}$ streptomycin (Sigma) and 10\% (v/v) fetal calf serum (Serum Laboratories International)). Cells were grown as a monolayer at a density of $10^{7}$ cells per $75 \mathrm{~mm}^{2}$ flask at 37 ${ }^{\circ} \mathrm{C}$ in $95 \%$ air and $5 \% \mathrm{CO}_{2}$, with medium changed every $48 \mathrm{~h}$. For passages, cells were detached with trypsin/EDTA (Life Technologies) at $37^{\circ} \mathrm{C}$, then washed in complete culture medium and replated.

\section{Induction of ER stress and isolation of extracellular vesicles}

Confluent T75 flasks of BeWo cells were treated with either growth medium containing tunicamycin (Sigma) or thapsigargin (Sigma) at the concentrations shown. DMSO was used as vehicle-only control. All media were made with FBS which had been centrifuged at $150,000 \mathrm{xg}$ overnight for depletion of extracellular vesicles. After $48 \mathrm{~h}$ the medium was collected from the cells and spun at $1500 \mathrm{xg}$ for $10 \mathrm{~min}$ at $4{ }^{\circ} \mathrm{C}$ to remove large cellular debris. The supernatant was spun for $1 \mathrm{~h}$ at $150,000 \mathrm{xg}$ at $4^{\circ} \mathrm{C}$. The resultant $\mathrm{EV}$ pellet was then resuspended in sterile PBS for further analysis. 


\section{Nanoparticle tracking analysis}

EV sizes and concentrations were measured using the NanoSight NS500 instrument equipped with a $405 \mathrm{~nm}$ laser (Malvern, UK), sCMOS camera and nanoparticle tracking analysis (NTA) software version 2.3, Build 0033 (Malvern, UK) as previously described [50]. Briefly, samples were automatically introduced into the sample chamber and the following script was used for EV measurements: PRIME, DELAY 5, CAPTURE 30, REPEAT 9. Samples were recorded using a camera level of 12 (camera shutter speed $15 \mathrm{~ms}$; camera gain 350) and NTA post-acquisition settings were optimised and kept constant between samples. Each video recording was analysed to give EV size and concentration measurements.

\section{Immunoblotting}

Immunoblotting was performed as described previously [51]. For cell lysates, protein concentration was determined using a BCA protein assay kit (Pierce) and equal amounts of protein $(10 \mu \mathrm{g})$ were loaded. For EV preparations, equal volumes of the resuspended EV pellets were loaded. Primary antibodies were purchased from Abcam ( $\beta$-actin (ab6276), HMGB1 (ab18256), syntenin (ab144267), Cited-2 (ab108345), total JNK (ab179461), phospho-JNK (ab179461) and Cell Signaling Technology (GRP78 (3183), CHOP (2985), cleaved PARP (9541), hsp70 (4872), histone H3 (4499), alix (2171), p-eIF2 $\alpha$ (9721).

\section{Protein arrays}

Protein arrays were performed using a Proteome Profiler Human Cell Stress Array Kit (R\&D Systems). Briefly, BeWo cell lysates were incubated with the arrays at $4^{\circ} \mathrm{C}$ overnight and proteins were detected according to the manufacturer's instructions. Dots were detected using $\mathrm{X}$-ray film and densitometric analysis was carried out using Image $\mathrm{J}$ software.

\section{Optiprep $^{\mathrm{TM}}$ density gradient centrifugation}

Separation of EVs according to their buoyant densities was carried out using the method of Tauro et al [23]. Briefly, EV pellets were isolated from the culture medium from three T75 flasks of confluent BeWo cells as described above. Pellets were pooled and resuspended in PBS $(500 \mu \mathrm{l})$ and overlaid onto $5-40 \%$ discontinuous iodixanol gradients (prepared from Optiprep ${ }^{\mathrm{TM}} 60 \%(\mathrm{w} / \mathrm{v})$ aqueous iodixanol, Axis-Shield PoC). Ultracentrifugation was carried out at $150,000 \mathrm{xg}$ (maximum) for $18 \mathrm{~h}$ at $4^{\circ} \mathrm{C}$. Fractions $(12 \times 1 \mathrm{ml})$ were collected manually and NTA was carried out to determine the EV concentration of each fraction. To determine the density of each fraction the refractive index was measured using a hand-held refractometer (Mettler Toledo) and converted to density using a the following equation:

$$
\rho=3.4713 \eta-3.6393
$$

where $\rho=$ density and $\eta=$ refractive index.

\section{Lactate dehydrogenase activity assay}

Cell death was assessed by measurement of lactate dehydrogenase $(\mathrm{LDH})$ release into the culture medium. LDH activity was determined using an LDH Cytotoxicity Colorimetric Assay Kit II (BioVision) according to the manufacturer's protocol. Briefly, culture medium was aspirated and spun at $3000 \mathrm{x}$ g for $2 \mathrm{~min}$ at room temperature and $10 \mu \mathrm{l}$ aliquots of the supernatant were transferred into a 96-well plate. LDH Reaction Mix (100 $\mu \mathrm{l})$ was added and after incubation for $30 \mathrm{~min}$ absorbance at $450 \mathrm{~nm}$ was measured using a FLUOstar Optima plate reader (BMG Labtech). Results were expressed as the percentage of total cellular LDH (measured by lysis of cells for $30 \mathrm{~min}$ prior to collection of culture supernatants) released into the culture medium.

\section{Statistics}

The data presented represent mean $\pm \mathrm{SEM}$ of at least three separate experiments. Differences between treatment groups were analysed by one-way ANOVA or two-tailed Student's t-test, as indicated, and a $P$-value of $<0.05$ was considered to be statistically significant.

\section{CONFLICTS OF INTEREST}

The authors declare no conflicts of interest.

\section{FUNDING}

This work was supported by MRC Programme Grant MR/J003360/1.

\section{REFERENCES}

1. Oakes SA, Papa FR. The role of endoplasmic reticulum stress in human pathology. Annu Rev Pathol Mech Dis. 2015; 10:173-94.

2. Tersey SA, Nishiki Y, Templin AT, Cabrera SM, Stull ND, Colvin SC, Evans-Molina C, Rickus JL, Maier B, Mirmira $\mathrm{RG}$. Islet $\beta$-cell endoplasmic reticulum stress precedes the onset of type 1 diabetes in the nonobese diabetic mouse model. Diabetes. 2012; 61:818-27.

3. Seimon TA, Nadolski MJ, Liao X, Magallon J, Nguyen M, Feric NT, Koschinsky ML, Harkewicz R, Witztum JL, Tsimikas S, Golenbock D, Moore KJ, Tabas I. Atherogenic lipids and lipoproteins trigger CD36-TLR2-dependent 
apoptosis in macrophages undergoing endoplasmic reticulum stress. Cell Metab. 2010; 12:467-82.

4. Sprenkle NT, Sims SG, Sanchez CL, Meares GP. Endoplasmic reticulum stress and inflammation in the central nervous system. Mol Neurodegener. 2017; 12:42.

5. Yung HW, Calabrese S, Hynx D, Hemmings BA, Cetin I, Charnock-Jones DS, Burton GJ. Evidence of placental translation inhibition and endoplasmic reticulum stress in the etiology of human intrauterine growth restriction. Am J Pathol. 2008; 173:451-462.

6. Yung HW, Atkinson D, Campion-Smith T, Olovsson M, Charnock-Jones DS, Burton GJ. Differential activation of placental unfolded protein response pathways implies heterogeneity in causation of early- and late-onset preeclampsia. J Pathol. 2014; 234:262-276.

7. Raposo G, Stoorvogel W. Extracellular vesicles: exosomes, microvesicles and friends. J Cell Biol. 2013; 200:373-383.

8. Sargent I, Dragovic RA, Tannetta D, Redman C. Extracellular vesicles in normal pregnancy and preeclampsia. In: P Harrison, C Gardiner, I Sargent (Eds), Extracellular vesicles in health and disease, Pan Stanford Publishing, Singapore. 2014, pp. 357-390.

9. Schaefer L. Complexity of danger: the diverse nature of damage-associated molecular patterns. J Biol Chem. 2014; 289:35237-35245.

10. Ma CY, Jiao YL, Zhang J, Yang QR, Zhang ZF, Shen YJ, Chen ZJ, Zhao YR. Elevated plasma level of HMGB1 is associated with disease activity and combined alterations with IFN-alpha and TNF-alpha in systemic lupus erythematosus. Rheumatol Int. 2012; 32:395-402.

11. Yan XX, Lu L, Peng WH, Wang LJ, Zhang Q, Zhang RY, Chen QJ, Shen WF. Increased serum HMGB1 level is associated with coronary artery disease in nondiabetic and type 2 diabetic patients. Atherosclerosis. 2009; 205:544-548.

12. Kang R, Lotze MT, Herbert JZ, Billiar TR, Tang D. Cell death and DAMPs in acute pancreatitis. Mol Med. 2014; 20:466-477.

13. Naruse $\mathrm{K}$, Sado $\mathrm{T}$, Noguchi $\mathrm{T}$, Tsunemi $\mathrm{T}$, Yoshida $\mathrm{S}$, Akasaka J, Koike N, Oi H, Kobayashi H. Peripheral RAGE (receptor for advanced glycation endproducts)-ligands in normal pregnancy and preeclampsia: novel markers of inflammatory response. J Reprod Immunol. 2012; 93:69-74.

14. Fukushima A, Kawahara H, Isurugi C, Syoji T, Oyama R, Sugiyama T, Horiuchi S. Changes in serum levels of heat shock protein 70 in preterm delivery and pre-eclampsia. $\mathrm{J}$ Obstet Gynaecol Res. 2005; 31:72-77.

15. Wikstrom AK, Ekegren L, Karlsson M, Wikstrom J, Bergenheim M, Akerud H. Plasma levels of S100B during pregnancy in women developing pre-eclampsia. Pregnancy Hypertens. 2012; 2:398-402.

16. Jiang R, Cai J, Zhu Z, Chen D, Wang J, Wang Q, Teng Y, Huang Y, Tao M, Xia A, Xue M, Zhou S, Chen AF. Hypoxic trophoblast HMGB1 induces endothelial cell hyperpermeability via the TLR-4/caveolin-1 pathway. J Immunol. 2014; 193:5000-5012.

17. Tskitishvili E, Sharentuya N, Temma-Asano K, Mimura K, Kinugasa-Taniguchi Y, Kanagawa T, Fukuda H, Kimura T, Tomimatsu T, Shimoya K. Oxidative stress-induced S100B protein from placenta and amnion affects soluble endoglin release from endothelial cells. Mol Hum Reprod. 2010; 16:188-199.

18. Buzas EI, Gyorgy B, Nagy G, Falus A, Gay S. Emerging role of extracellular vesicles in inflammatory diseases. Nat Rev Rheumatol. 2014; 10:356-364.

19. Schiller M, Heyder P, Ziegler S, Niessen A, Classen L, Lauffer A, Lorenz HM. During apoptosis HMGB1 is translocated into apoptotic cell-derived membraneous vesicles. Autoimmunity. 2010; 46:342-346.

20. Clayton A, Turkes A, Navabi H, Mason MD, Tabi Z. Induction of heat shock proteins in B-cell exosomes. J Cell Sci. 2005; 118:3631-3638.

21. Feranchak AP, Lewis MA, Kresge C, Sathe M, Bugde A, Luby-Phelps K, Antich PP, Fitz JG. Initiation of purinergic signalling by exocytosis of ATP-containing vesicles in liver epithelium. J Biol Chem. 2010; 285:8138-8147.

22. Kakazu E, Mauer AS, Yin M, Malhi H. Hepatocytes release ceramide-enriched pro-inflammatory extracellular vesicles in an IRE1 $\alpha$-dependent manner. J Lipid Res. 2016; 57:233-245.

23. Tauro BJ, Greening DW, Mathias RA, Ji H, Mathivanan S, Scott AM, Simpson RJ. Comparison of ultracentrifugation, density gradient separation, and immunoaffinity capture methods for isolating human colon cancer cell line LIM1863-derived exosomes. Methods. 2012; 56:293-304.

24. Tauro BJ, Mathias RA, Greening DW, Gopal SK, Ji H, Kapp EA, Coleman BM, Hill AF, Kusebauch U, Hallows JL, Shteynberg D, Moritz RL, Zhu HJ, Simpson RJ. Oncogenic H-Ras reprograms Madin-Darby canine kidney (MDCK) cell-derived exosomal proteins following epithelial-mesenchymal transition. Mol Cell Proteomics. 2013; 12:2148-2159.

25. Santos CX, Tanaka LY, Wosniak J Jr, Laurindo FR. Mechanisms and implications of reactive oxygen species generation during the unfolded protein response: roles of endoplasmic reticulum oxidoreductases, mitochondrial electron transport, and NADPH oxidase. Antioxid Redox Signal. 2009; 11:2409-2427.

26. Luo SS, Ishibashi O, Ishikawa G, Ishikawa T, Katayama A, Mishima T, Takizawa T, Shigihara T, Goto T, Izumi A, Ohkuchi A, Matsubara S, Takeshita T, Takizawa T. Human villous trophoblasts express and secrete placenta-specific microRNAs into maternal circulation via exosomes. Biol Reprod. 2009; 81:717-729.

27. Holder BS, Tower CL, Forbes K, Mulla MJ, Aplin JD, Abrahams VM. Immune cell activation by trophoblastderived microvesicles is mediated by syncytin 1 . Immunology. 2012; 136:184-191. 
28. Al-Nasiry S, Spitz B, Hanssens M, Luyten C, Pijnenborg R. Differential effects of syncytialization and apoptosis on BeWo and JEG-3 choriocarcinoma cells. Hum Reprod. 2006; 21:193-201.

29. Burton GJ, Yung HW. Endoplasmic reticulum stress in the pathogenesis of early-onset pre-eclampsia. Pregnancy Hypertens. 2011; 1:72-78.

30. Eguchi A, Mulya A, Lazic M, Radhakrishnan D, Berk MP, Povero D, Gornicka A, Feldstein AE. Microparticles release by adipocytes act as "find-me" signals to promote macrophage migration. PLoS One. 2015; 10:e0123110.

31. Zhu L, Zhang Z, Zhang L, Shi Y, Qi J, Chang A, Gao J, Feng Y, Yang X. HMGB1-RAGE signalling pathway in severe preeclampsia. Placenta. 2015; 36:1148-1152.

32. Peracoli JC, Bannwart-Castro CF, Romao M, Weel IC, Ribeiro VR, Borges VTM, Rudge MV, Witkin SS, Peracoli MT. High levels of heat shock protein 70are associated with pro-inflammatory cytokines and may differentiate earlyfrom late-onset preeclampsia. J Reprod Immunol. 2013; 100:129-134.

33. Bakker WW, Donker RB, Timmer A, van Pampus MG, van Son WJ, Aarnoudse JG, van Goor H, Niezen-Koning KE, Navis G, Borghuis T, Jongman RM, Faas MM. Plasma hemopexin activity in pregnancy and preeclampsia. Hypertens Pregnancy. 2007; 26:227-239.

34. Artunc-Ulkumen B, Guvenc Y, Goker A, Gozukara C. Maternal serum S100-B, PAPP-A and IL-6 levels in severe preeclampsia. Arch Gynecol Obstet. 2015; 292:97-102.

35. Qiu C, Hevner K, Enquobahrie DA, Williams MA. A casecontrol study of maternal blood mitochondrial DNA copy number and preeclampsia risk. Int J Mol Epidemiol Genet. 2012; 3:237-244.

36. Vega VL, Rodriguez-Silva M, Frey T, Gehrmann M, Diaz JC, Steinem C, Multhoff G, Arispe N, De Maio A. Hsp70 translocates into the plasma membrane after stress and is released into the extracellular environment in a membraneassociated form that activates macrophages. J Immunol 2008; 180:4299-4307.

37. Wickman GR, Julian L, Mardilovich K, Schumacher S, Munro J, Rath N, Zander SA, Mleczak A, Sumpton D, Morrice N, Bienvenut WV, Olson MF. Blebs produced by actin-myosin contraction during apoptosis release damageassociated molecular pattern proteins before secondary necrosis occurs. Cell Death Differ. 2013; 20:1293-1305.

38. Hwang C, Sinskey AJ, Lodish HF. Oxidized redox state of glutathione in the endoplasmic reticulum. Science. 257; 5076:1496-1502.

39. Ding W, Yang L, Zhang M, Gu Y. Reactive oxygen speciesmediated endoplasmic reticulum stress contributes to aldosterone-induced apoptosis in tubular epithelial cells. Biochem Biophys Res Com. 2012; 418:451-456.

40. Lin H, Liu $\mathrm{X}$, Yu J, Hua F, Hu Z. Antioxidant $\mathrm{N}$-acetylcysteine attenuates hepatocarcinogenesis by inhibiting ROS/ER stress in TLR2 deficient mice. PLoS One. 2013; 8:e74130.

41. Kranc KR, Schepers H, Rodrigues NP, Bamforth S, Villadsen E, Ferry H, Bouriez-Jones T, Sigvardsson M, Bhattacharya S, Jacobsen SE, Enver T. Cited2 is an essential regulator of adult hematopoietic stem cells. Cell Stem Cell. 2009; 5:659-665.

42. Moreau JL, Artap ST, Shi HJ, Chapman G, Leone G, Sparrow DB, Dunwoodie SL. Cited2 is required in trophoblasts for correct placental capillary patterning. Dev Biol. 2014; 392:62-79.

43. Gu H, Yu JW, Dong DY, Zhou Q, Wang JY, Fang SY, Yang PX. High glucose-repressed CITED2 expression through miR-200b triggers the unfolded protein response and endoplasmic reticulum stress. Diabetes. 2016; 65:149-163.

44. Ostman A, Yang Q, Tonks NK. Expression of DEP-1, a receptor-like protein-tyrosine-phosphatase, is enhanced with increasing cell density. Proc Natl Acad Sci U S A. 1994; 91:9680-9684.

45. Cancela ML, Hu B, Price PA. Effect of cell density and growth factors on matrix GLA protein expression by normal rat kidney cells. J Cell Physiol 1997; 171:125-134.

46. Chan JY, Luzuriaga J, Maxwell EL, West PK, Bensellam M, Laybutt DR. The balance between adaptive and apoptotic unfolded protein responses regulates beta-cell death under ER stress conditions through XBP1, CHOP and JNK. Mol Cell Endocrinol. 2015; 413:189-201.

47. Shimizu T, Yamakuchi M, Biswas KK, Aryal B, Yamada S, Hashiguchi T, Maruyama I. HMGB1 is secreted by 3T3L1 adipocytes through JNK signaling and the secretion is partially inhibited by adiponectin. Obesity. 2016; 24:1913-1921.

48. Gan LT, Van Rooyen DM, Koina ME, McCuskey RS, Teoh NC, Farrell GC. Hepatocyte free cholesterol lipotoxicity results from JNK1-mediated mitochondrial injury and is HMGB1 and TLR4-dependent. J Hepatol. 2014; 61:1376-1384.

49. Hasnain SZ, Lourie R, Das I, Chen AC, McGuckin MA. The interplay between endoplasmic reticulum stress and inflammation. Immunol Cell Biol. 2012; 90:260-270.

50. Dragovic RA, Collett GP, Hole P, Ferguson DJ, Redman $\mathrm{CW}$, Sargent IL, Tannetta D. Isolation of syncytiotrophoblast microvesicles and exosomes and their characterisation by multicolour flow cytometry and fluorescence nanoparticle tracking analysis. Methods. 2015; 87:64-74.

51. Collett GP, Goh XF, Linton EA, Redman CW, Sargent IL. RhoE is regulated by cyclic AMP and promotes fusion of human BeWo choriocarcinoma cells. PLoS One. 2012; 7:e30453. 